\title{
K-edge subtraction imaging using a pixellated energy-resolving detector
}

\author{
Silvia Pani ${ }^{* a}$, Sarene C. Saifuddin ${ }^{\mathrm{a}}$, Christiana Christodoulou ${ }^{\mathrm{b}}$, Matthew Veale ${ }^{\mathrm{c}}$, Paul Seller ${ }^{\mathrm{c}}$, Robert \\ D. Speller ${ }^{\mathrm{b}}$, Matthew Wilson ${ }^{\mathrm{c}}$, James W. Scuffham ${ }^{\mathrm{a}, \mathrm{d}}$ \\ ${ }^{\mathrm{a}}$ Department of Physics, University of Surrey, Guildford, UK; \\ ${ }^{\mathrm{b}}$ Dept. of Medical Physics and Bioengineering, University College London, UK;

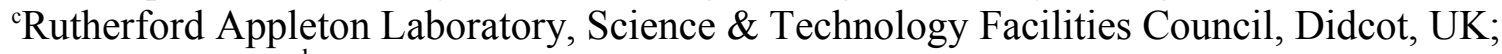 \\ ${ }^{\mathrm{d}}$ Royal Surrey County Hospital NHS Foundation Trust.
}

\begin{abstract}
This paper presents preliminary work aimed at assessing the feasibility of K-edge subtraction imaging using the spectroscopic information provided by a pixellated energy-resolving Cadmium Zinc Telluride detector, having an active area of 20x20 pixels $250 \mu \mathrm{m}$ in size. Images of a test object containing different amounts of Iodine-based contrast agent were formed above and below the K-edge of Iodine $(33.2 \mathrm{keV})$ by integrating, pixel by pixel, different windows of the spectrum. The results show that the optimum integration window for details $1-2 \mathrm{~mm}$ in diameter is between $2 \mathrm{keV}$ and 5 $\mathrm{keV}$. Concentrations of down to $50 \mu \mathrm{g}$ Iodine $/ \mathrm{ml}$ were detected in a 1-mm diameter tube with an entrance dose of 100 $\mu \mathrm{Gy}$.
\end{abstract}

Keywords: K-edge subtraction, pixellated spectroscopic detectors, Cadmium Zinc Telluride.

\section{INTRODUCTION}

K-edge subtraction (KES) imaging is typically implemented at synchrotron sources, where the possibility of finely tuning the beam energy allows two images to be taken at energies immediately below and immediately above the K-edge of a contrast agent, thus maximizing the differences in absorption in regions where the contrast agent is present, and minimizing the differences in other regions. Several works [1-5] have proven the effectiveness of the technique in background removal and quantitative characterization.

However, the main limitation of the technique lies in the need for monochromatic, energy-tunable beams, which makes it difficult to implement it outside synchrotron sources, although some work [4,5] has been carried out to identify suitable combinations of polychromatic spectra. In this case, the contrast achieved is lower than that achieved with synchrotron radiation as the maximum difference in the attenuation coefficient of the contrast agent above and below the K-edge is not fully exploited.

We propose an alternative approach, based on the use of a pixellated spectroscopic detector and of a polychromatic beam from a conventional source. The spectroscopic capability of the detector allows the selection of arbitrary energy windows to be integrated to form images above and below the K-edge. This allows better contrast resolution than standard methods used for conventional sources, still keeping the high degree of accessibility resulting from the use of a laboratory source as opposed to synchrotron radiation.

Another advantage of the method proposed here is that the two images above and below the K-edge are acquired simultaneously. This allows dose reduction by a factor of 2 , and ensures images free from motion artifacts.

\footnotetext{
*s.pani@surrey.ac.uk; phone +44 1483682276; fax +44 1483686781
} 


\section{METHODS}

\section{Experimental setup}

The experimental setup consisted of a W-anode X-ray source, a pixellated energy-resolving Cadmium Zinc Telluride detector and a sample containing different amounts of Iodine-based contrast agent.

The object was placed at $53 \mathrm{~cm}$ from the source, and the detector was placed at $68.5 \mathrm{~cm}$ from the source.

The X-ray source (Hamamatsu L6371-MFX) was operated at $50 \mathrm{kVp}$ and $10 \mu \mathrm{A}$; the beam was filtered with $2 \mathrm{~mm} \mathrm{Al}$ in order to remove low-energy components of the beam not useful for image formation. In this way, the maximum of the beam spectrum was around $30 \mathrm{keV}$, very close to the K-edge of Iodine $(33.2 \mathrm{keV})$. A typical beam spectrum for these settings [6], with the Iodine K-edge marked, is shown in Figure 1.

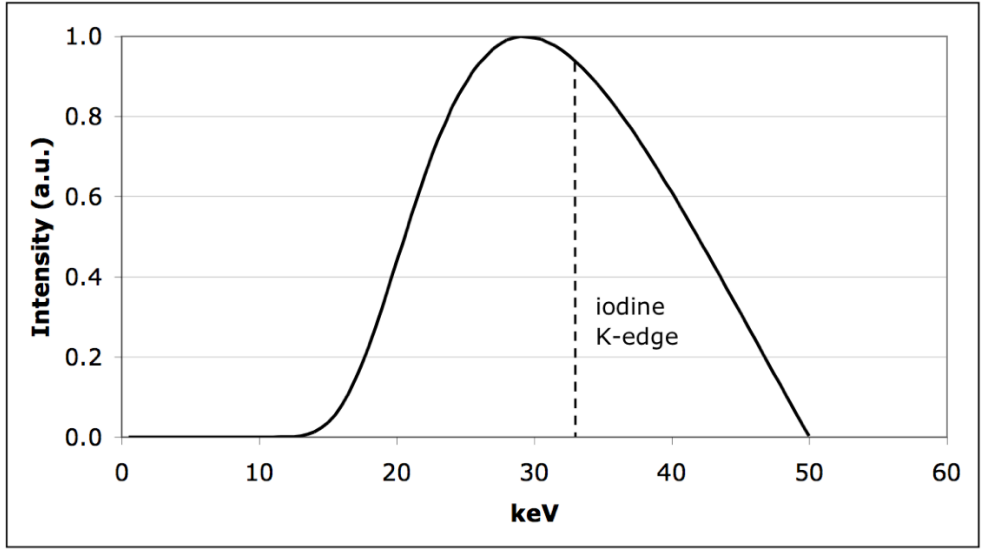

Figure 1. Typical X-ray spectrum for the beam settings used [6]. The K-edge of Iodine (33.2 keV) is marked.

The test object consisted of a Perspex slab, $6 \mathrm{~mm}$ in thickness, with cylindrical holes $3 \mathrm{~mm}, 2 \mathrm{~mm}$ and $1 \mathrm{~mm}$ in diameter, respectively. The axes of the cylinders were kept orthogonal to the beam direction. The holes were filled with Iodinebased commercial contrast agent (Niopam 150, Bracco). The contrast agent was used undiluted (giving an effective concentration of $150 \mu \mathrm{g}$ Iodine $/ \mathrm{ml}$ ) or diluted, to give concentrations of $100 \mu \mathrm{g}$ Iodine $/ \mathrm{ml}, 75 \mu \mathrm{g}$ Iodine $/ \mathrm{ml}, 50 \mu \mathrm{g}$ Iodine/ml, respectively.

The detector used was a 20x20 pixel Cadmium Zinc Telluride (Redlen) with 250x250 $\mu \mathrm{m}^{2}$ pixels, for a total active area of $0.5 \times 0.5 \mathrm{~cm}^{2}$ and equipped with custom readout electronics [7]. The detector was read out at a rate of 21000 frames per second.

In order to avoid loss of linearity at high photon fluxes, that would have affected image contrast, different statistics were achieved by keeping the tube current constant and altering the integration time.

For the acquisition of object regions bigger than the detector area, the object was scanned in front of the detector using a remote-controlled linear stage (Micos VT-80 with SMC controller); the images acquired at different steps were combined off-line.

\section{Image reconstruction and analysis}

The data processing software used [8] provided, for each pixel, a spectrum of the photons detected by that pixel. An example is shown in Figure 2, where the Iodine K-edge is also highlighted.

The images obtained above and below the K-edge were combined into a log-subtracted image $S$, obtained as follows: 


$$
S(i, j)=\ln \left(I_{\text {high }}^{\text {bg }} / I_{\text {high }}(i, j)\right)-\ln \left(I_{\text {low }}^{\text {bg }} / I_{\text {low }}(i, j)\right)
$$

where $I_{\text {high }}, I_{\text {low }}$ are the images obtained above and below the K-edge, respectively, $I_{\text {high }}^{b g}$ is the average background signal for the image above the K-edge and $I_{l o w}^{b g}$ is the average background signal for the image below the K-edge.

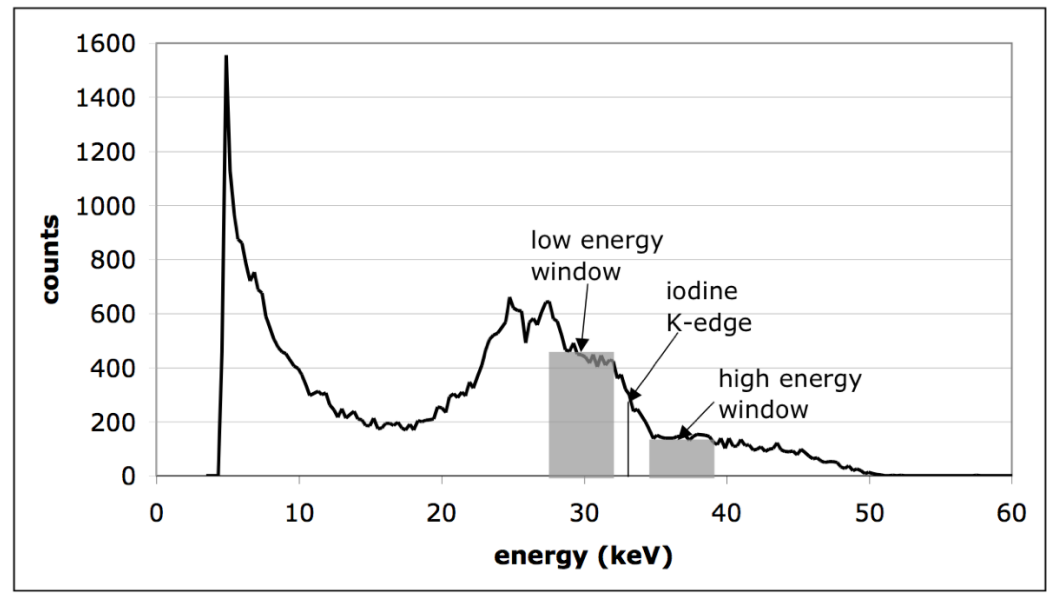

Figure 2. Example of a transmitted spectrum after $2 \mathrm{~mm}$ of Iodine-based contrast agent. The Iodine K-edge and two selections of low energy window and high energy window are highlighted.

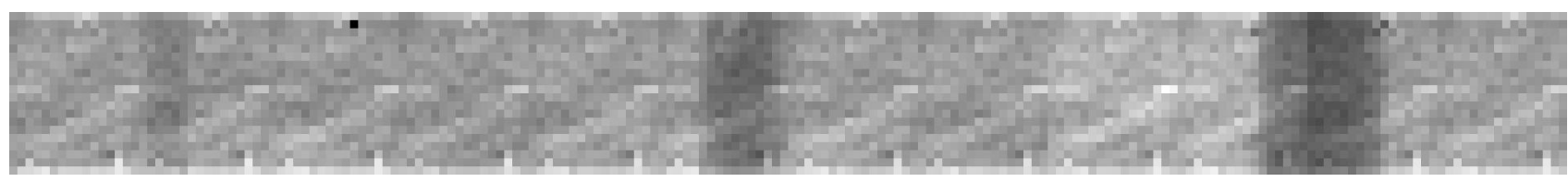

(a)

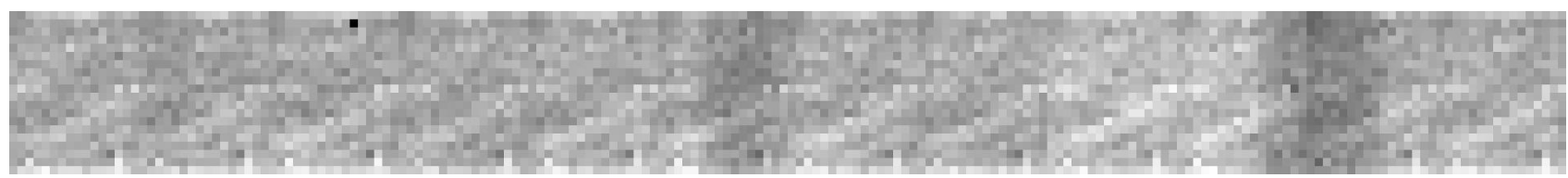

(b)

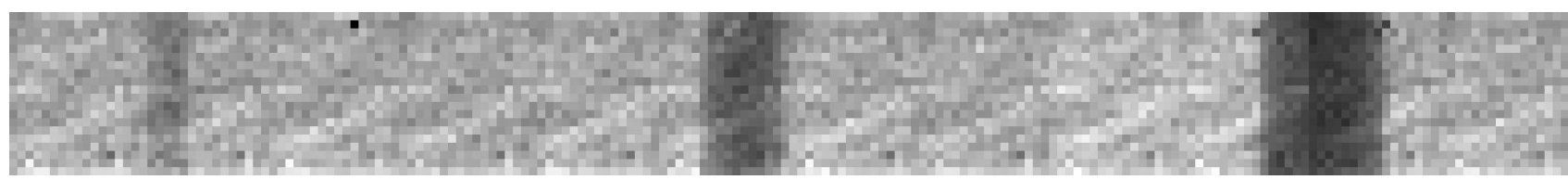

(c)

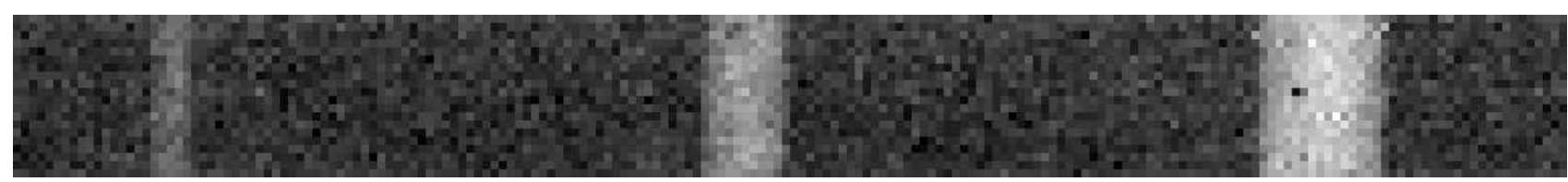

(d)

Figure 3. Images of a test object with tubes containing a Iodine-based contrast agent $(1 \mathrm{~mm}, 2 \mathrm{~mm}$ and $3 \mathrm{~mm}$ in diameter), obtained: (a) From integration of the whole spectrum; (b) from integration of a 2-keV energy band below the K-edge of Iodine; (c) from integration of a 2-keV energy band above the K-edge of Iodine; (d) from logarithmic subtraction of (b) and (c). 
An example is shown in Figure 3, showing an image obtained by integrating the whole spectrum (simulating an image obtained with a non-spectroscopic detector), images obtained by integrating $2-\mathrm{keV}$ energy windows below and above the K-edge of Iodine, respectively, and the log-subtracted image.

The overall visibility of each detail was quantified using the contrast-to-noise ratio, defined as

$$
C N R=\frac{D-B}{\sigma_{B}}
$$

where $D$ is the average signal in the detail region (in this case the central region of the cylinder), $B$ is the average signal in the background and $\sigma_{\mathrm{B}}$ is the standard deviation associated to the background region. Images obtained by integrating a narrow energy window will feature maximum contrast but large noise; images obtained by integrating a wide energy window will feature high statistics, and hence low noise, but poorer contrast.

\section{RESULTS}

\section{Effect of the width of the integration windows}

Figure 4 shows log-subtracted images of the same object shown in Figure 3, obtained from images with different window width, proving the higher effectiveness for background removal of the narrow energy windows.

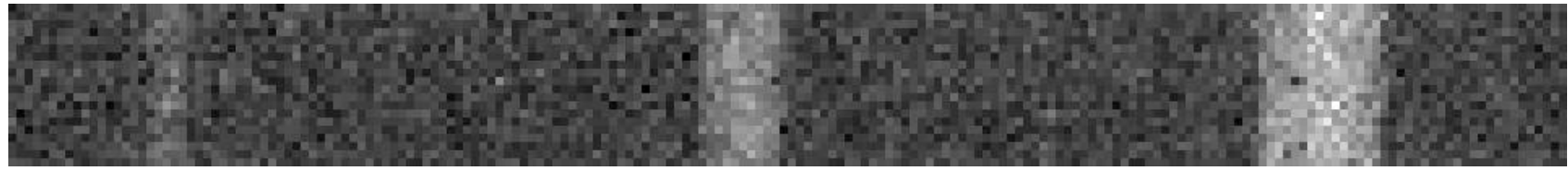

(a)

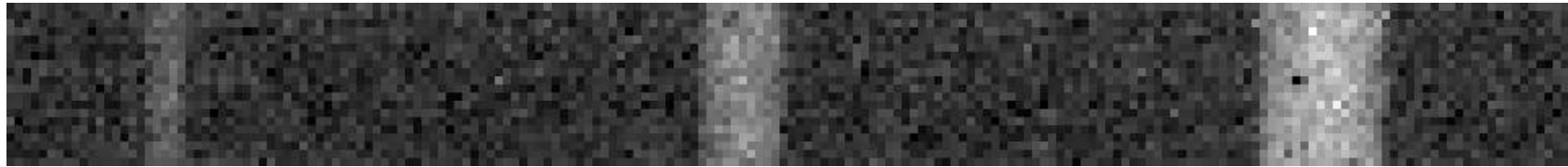

(b)

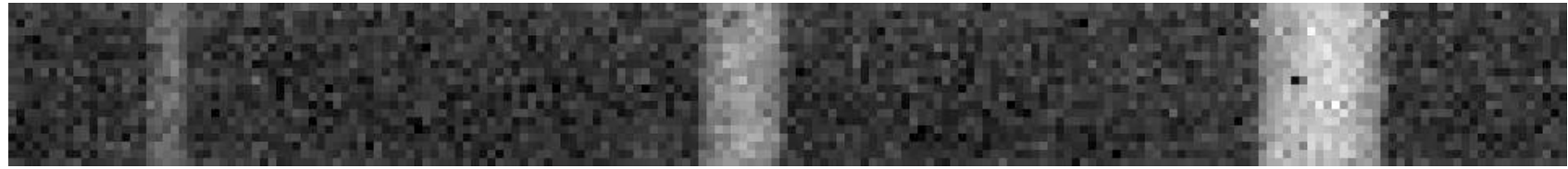

(c)

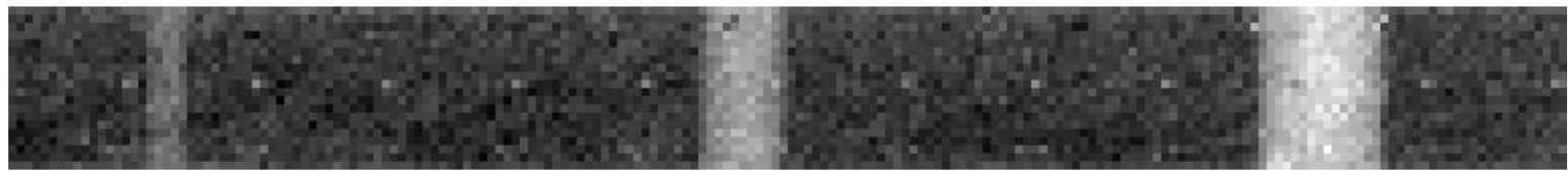

(d)

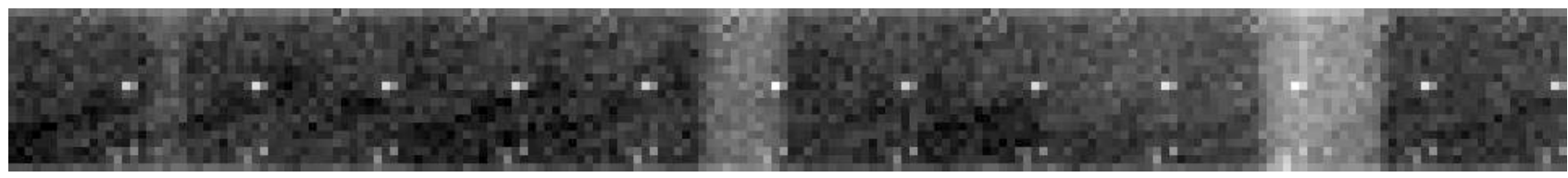

(e)

Figure 4. Log-subtracted images for the object shown in Figure 3. The integration band for the images above and below the K-edge are: (a) $0.5 \mathrm{keV}$; (b) $1 \mathrm{keV}$; (c) $2 \mathrm{keV}$; (d) $5 \mathrm{keV}$; (e) $10 \mathrm{keV}$. 
This is confirmed by the data plotted in figure 5, showing profiles across the 1-mm and the 2-mm tubes, respectively, for different integration windows. The Iodine concentration was $100 \mu \mathrm{g} / \mathrm{ml}$ and the entrance dose on the sample surface was $100 \mu \mathrm{Gy}$. The corresponding contrast-to-noise ratio is shown in Table 1 .

In both cases, a decrease in contrast is observed when moving from the $0.5 \mathrm{keV}$ window down to the $10 \mathrm{keV}$. In the latter case, the profile of the detail is poorly distinguishable. On the other hand, the low statistics contributing to the narrower energy bands causes the noise to be significantly higher in these cases.

This causes the contrast-to-noise ratio to have a maximum between 2 and $5 \mathrm{keV}$, as shown in Table 1 .

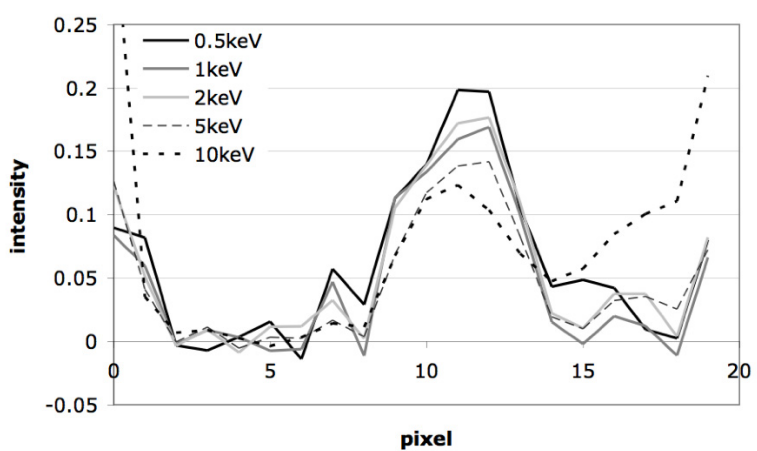

(a)

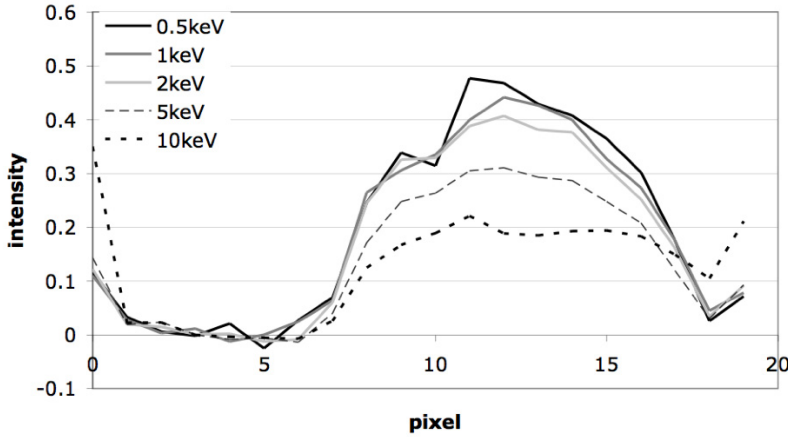

(b)

Figure 5. Comparison between the profiles across the detail for the $1 \mathrm{~mm}$ detail (a) and the $2 \mathrm{~mm}$ detail (b) filled with a concentration of $100 \mu \mathrm{g} / \mathrm{ml}$ Iodine and imaged at $100 \mu \mathrm{Gy}$.

Table 1. Comparison between the contrast-to-noise ratio for the data shown in Figure 5.

\begin{tabular}{ccc}
$\begin{array}{c}\text { Energy window } \\
(\mathbf{k e V})\end{array}$ & $\begin{array}{c}\text { CNR - } \\
\text { 1 mm tube }\end{array}$ & $\begin{array}{c}\text { CNR - } \\
\text { 2 mm tube }\end{array}$ \\
\hline \hline 0.5 & 13 & 56 \\
1 & 26 & 104 \\
2 & 58 & 121 \\
5 & 83 & 103 \\
10 & 13 & 34 \\
\hline
\end{tabular}

\subsection{Effect of contrast agent concentration}

Figures 6 shows images of the 1-mm tube obtained using a $5-\mathrm{keV}$ wide energy window at the minimum dose allowing visibility for each concentration.

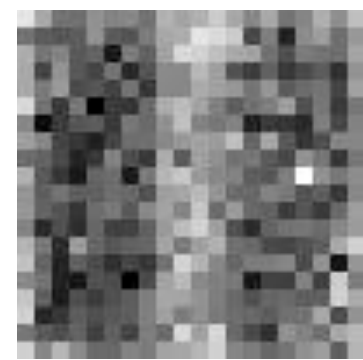

(a) $50 \mu \mathrm{g} / \mathrm{ml}$, $100 \mu \mathrm{Gy}$

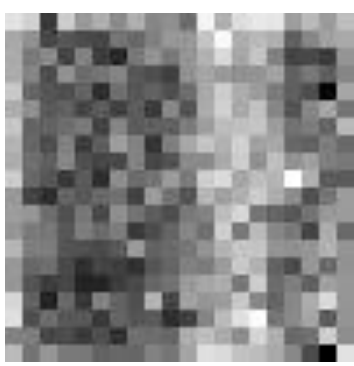

(b) $75 \mu \mathrm{g} / \mathrm{ml}$, $100 \mu \mathrm{Gy}$

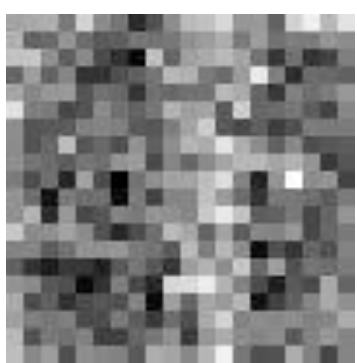

(c) $100 \mu \mathrm{g} / \mathrm{ml}$, $25 \mu \mathrm{Gy}$

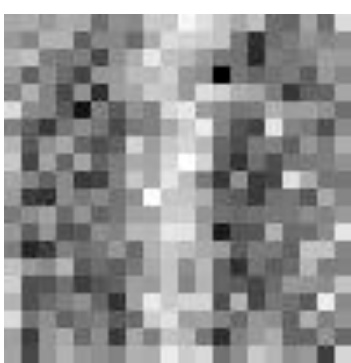

(d) $150 \mu \mathrm{g} / \mathrm{ml}$, $25 \mu \mathrm{Gy}$

Figure 6. Images of the 1-mm tube acquired at the minimum dose allowing detection for different concentrations of the contrast agent. 
The minimum entrance dose allowing detection of the $1-\mathrm{mm}$ tube filled with concentrations of $50 \mu \mathrm{g}$ Iodine/ml and 75 $\mu \mathrm{g}$ Iodine $/ \mathrm{ml}$ is $100 \mu \mathrm{Gy}$, and it decreases to $25 \mu \mathrm{Gy}$ for concentrations of $100 \mu \mathrm{g}$ Iodine $/ \mathrm{ml}$ and higher; the 2-mm tube was visible at all concentrations with all doses achievable with the current setup. Lower doses could not be achieved due to instability of the X-ray source below $10 \mu \mathrm{A}$.

Figure 7 shows the contrast-to-noise ratio as a function of the entrance dose to the sample for the different concentrations.

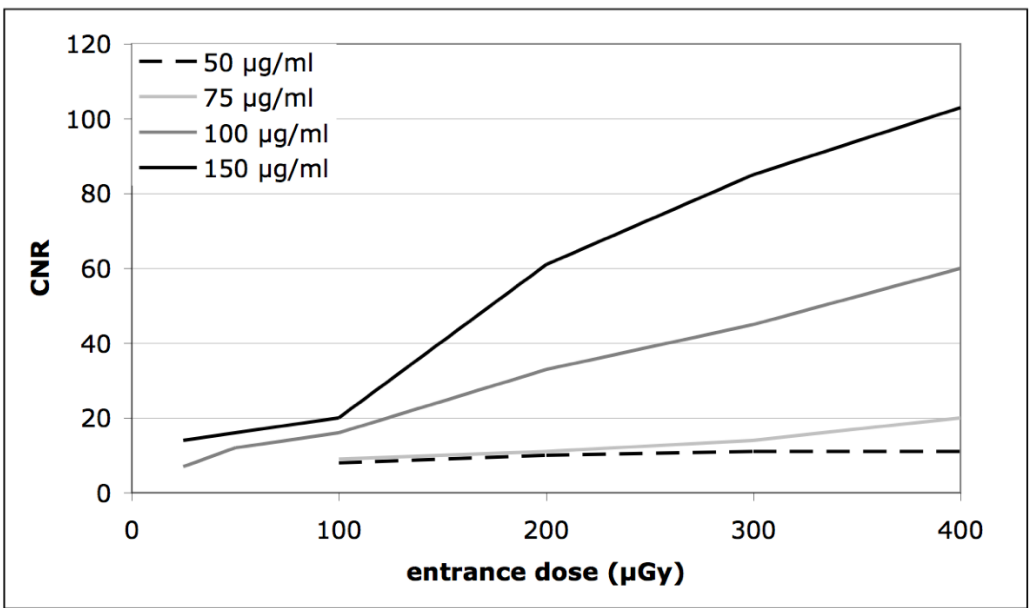

Figure 7. Contrast-to-noise ratio as a function of entrance dose for the 1-mm tube filled with different concentration of contrast agent.

\section{DISCUSSION}

The images in Figure 4 show that narrower energy windows allow better background removal than wider energy windows. This is because the attenuation coefficients of the background can be considered as nearly constant on the two side of the K-edge. In general, for a sufficiently narrow energy window, the intensities transmitted behind a detail of thickness $t$ and a background of thickness $L-t$ can be written as

$$
\begin{gathered}
I_{\text {low }}=I_{0}^{\text {low }} e^{-\mu_{b}^{\text {low }}(L-t)} e^{-\mu_{d}^{\text {low }} t} \\
I_{\text {high }}=I_{0}^{\text {high }} e^{-\mu_{b}^{\text {high }}(L-t)} e^{-\mu_{d}^{\text {high }} t}
\end{gathered}
$$

for the energy windows below and above the K-edge, respectively.

Here:

- $I_{0}{ }^{\text {low }}$ and $I_{0}{ }^{\text {high }}$ are the entrance photon intensity below and above the K-edge, respectively;

- $\mu_{b}^{\text {low }}$ and $\mu_{b}^{\text {high }}$ are the average attenuation coefficients of the background below and above the K-edge, respectively;

- $\mu_{d}^{\text {low }}$ and $\mu_{d}^{\text {high }}$ are the average attenuation coefficients of the detail below and above the K-edge, respectively.

The log-subtracted signal at the same point will be

$$
S=\left(\mu_{b}^{\text {high }}-\mu_{b}^{\text {low }}\right)(L-t)+\left(\mu_{d}^{\text {high }}-\mu_{d}^{\text {low }}\right) t
$$

Only if the attenuation coefficient of the background can be assumed to be constant above and below the K-edge the first term will cancel and the log-subtracted image has no dependence upon the background. This doesn't apply to wider energy windows, for which this assumption doesn't hold.

The data shown in Figure 5 and Table 1 show that the optimum energy window to ensure maximum contrast-to-noise ratio is between $2 \mathrm{keV}$ and $5 \mathrm{keV}$. This is because narrower energy windows, although ensuring maximum contrast by 
exploiting the maximum difference in the attenuation coefficients of the contrast agent below and above the K-edge, give rise to lower statistics, and hence higher noise. On the other hand, a wide energy window ensures high statistics but is affected by a strong decrease in contrast.

Due to the lower intrinsic contrast of the 1-mm tube, this detail has a better CNR when using a wider energy band, allowing the exploitation of higher statistics.

As expected, the limit detectable concentration is higher for the 1-mm detail than for the 2-mm detail. However, the doses involved in the present study are much lower than typical doses for imaging with conventional systems. Further dose reduction may be achieved by further narrowing the beam spectrum around the K-edge of the contrast agent, for instance by using a thicker Aluminium filter to reduce the low-energy components of the beam and a lower kilovoltage to remove the higher energies. Moreover, it must be pointed out that standard K-edge subtraction procedures need two separate acquisitions (one above the K-edge and one below the K-edge), thus requiring higher doses than other radiological procedures. As, in this case, the two images are obtained simultaneously, the technique is likely to allow significantly lower doses than conventional K-edge procedures.

The technique has great potential for use in angiography. In particular, the assessment of angiogenesis within and around a lesion could provide information about its malignancy, thus increasing the specificity of the diagnosis. This is particularly important in mammography. This imaging procedure normally relies on the use of low-energy X-ray spectra (from tubes with Molybdenum, Rhodium and, more rarely, Tungsten anodes operated between $25 \mathrm{kVp}$ and $35 \mathrm{kVp}$ ) in order to achieve detectable contrast between tumour and fibroglandular tissue. This need would be removed by the use of a contrast agent with a K-edge at higher energy. Besides Iodine, used for the present study, the use of Gadolinium (Kedge at $50.2 \mathrm{keV}$ ) could be addressed. Due to the thickness of the sensor $(2 \mathrm{~mm})$, its efficiency is expected to be close to $100 \%$ up to $70 \mathrm{keV}$, making it suitable for use at higher energies than those used for the present study.

\section{CONCLUSIONS AND FUTURE WORK}

This paper presented preliminary results on iodine K-edge subtraction imaging based on the use of a pixellated spectroscopic detector and on integration of the spectrum across different energy windows below and above the K-edge.

The technique proved effective in background removal. The choice of the optimum energy window width was discussed, and it was shown that, for the details imaged, it lies between $2 \mathrm{keV}$ and $5 \mathrm{keV}$, in order to guarantee a good compromise between statistics and contrast. However, as the integration of the energy windows is done off-line, it is expected that different images could be obtained from the same data set in order to fine-tune the visibility of different details.

The main advantage of the technique compared to standard K-edge subtraction procedures lies in the possibility of acquiring a single image, instead of the two normally needed for K-edge subtraction imaging. This implies reduced dose and removes any artifacts that may result from patient motion, and makes the technique suitable for in vivo angiography.

Future work will include optimization of the X-ray spectrum, in order to further reduce the energy components not contributing to image formation and hence reducing the dose.

Algorithms for the extraction of quantitative information from images [refs] will be developed and tested on different energy windows. For the reasons discussed when addressing the background subtraction capability for different energy windows, it can be expected that these algorithms will be less effective with wider energy windows.

Finally, methods for assessing the uptake and washout of contrast agent, through the use of dynamic test objects, will be evaluated.

\section{ACKNOWLEDGMENTS}

This work has been done within the EPSRC-funded HEXITEC collaboration (grant no. EP/H046577/1).

S. Saifuddin is sponsored by the Ministry of Health of Malaysia.

C. Christodoulou is sponsored by EPSRC. 


\section{REFERENCES}

[1] Baldelli, P., Bravin, A., Di Maggio, C., Gennaro, G., Sarnelli, A., Taibi, A., Gambaccini, M. "Evaluation of the minimum iodine concentration for contrast-enhanced subtraction mammography". Phys Med Biol 51, 4233-4251 (2006).

[2] Schültke, E., Fiedler, S., Nemoz, C., Ogieglo, L., Kelly, M.E., Crawford, P., Esteve, F., Brochard, T., Renier, M., Requardt, H., Le Duc, G., Juurlink, B., Meguro, K. "Synchrotron-based intra-venous K-edge digital subtraction angiography in a pig model: a feasibility study". Eur J Radiol 73, 677-681 (2010).

[3] Peterzol, A., Bravin, A., Coan, P., Elleaume, H. "Performance of the K-edge digital subtraction angiography imaging system at the European synchrotron radiation facility". Radiat Prot Dosimetry 117, 44-49 (2005).

[4] Skarpathiotakis, M., Yaffe, M.J., Bloomquist, A.K., Rico, D., Muller, S., Rick, A., Jeunehomme, F. "Development of contrast digital mammography". Med Phys 29, 2419-2426 (2002).

[5] Lewin, J.M., Isaacs,P.K., Vance, V. and Larke, F.J. "Dual-energy contrast-enhanced subtraction mammography: feasibility" Radiology 229, 261-268 (2003).

[6] Cranley, K., Gilmore, B.J., Fogarty, G.W.A., Desponds, L. "Catalogue of Diagnostic X-Ray Spectra \& Other Data”. IPEM Report 78, on CD-ROM.

[7] Jones, L., Seller, P., Wilson, M., Hardie, A. "HEXITEC ASIC—a pixellated readout chip for CZT detectors". Nucl Instr Meth A 604, 34-37 (2009).

[8] Sochi, T. "High throughput software for powder diffraction and its application to heterogeneous catalysis". PhD Dissertation, University of London (2010). 